\title{
ELIGE EDUCAR, UN PUNTO DE ENCUENTRO ENTRE ACTORES PARA MEJORAR LA CALIDAD DE LA EDUCACIÓN EN CHILE
}

\author{
María Paz Medeiros, Gabriel Gutiérrez, \\ Hernán Hochschild, Rafael Lira ${ }^{1}$
}

\begin{abstract}
RESUMEN
El siguiente documento sistematiza el trabajo de la iniciativa "Elige Educar", que constituye un intento por revertir los bajos niveles de valoración social de los profesionales de la educación en Chile. Para esto, enuncia datos del contexto que permiten entender su justificación y explica, en términos generales, su acción.

Asimismo, revisa con especial énfasis el trabajo que Elige Educar ha desarrollado con una serie de universidades, tanto en la atracción de estudiantes talentosos por medio de becas, como por la campaña de comunicaciones que desarrolla el programa.

Finalmente da cuenta de los primeros avances y concluye dando a conocer los desafíos de la iniciativa en términos de su trabajo con las facultades de educación.
\end{abstract}

Palabras clave: profesores, valoración social, formación inicial docente, efectividad docente

\section{ELIGE EDUCAR (CHOOSE TEACHING), A MEETING POINT FOR ACTORS TO IMPROVE THE QUALITY OF EDUCATION IN CHILE}

\section{ABSTRACT}

This document systematizes the work of the public private initiative "Elige Educar" (Choose teaching), which constitutes an innovative social entrepreneurship aimed to reverse the low levels of social valuation of teachers in Chile. To that end, it sets a context for understanding the rationale and explains its action in general terms.

Likewise, it specially reviews the work Elige Educar has developed with a number of Universities, both in attracting talented students through scholarships and through communications campaigns developed by the initiative.

Finally, it presents challenges in terms of work in schools of education of different Universities.

Keywords: teachers, social valuation, teacher initial training, teacher effectiveness

1 Centro de Políticas Públicas, Pontificia Universidad Católica de Chile. Santiago. Chile. Contacto: hhochschild@eligeeducar.cl 


\section{ELIGE EDUCAR, UN PUNTO DE ENCUENTRO ENTRE ACTORES PARA MEJORAR LA CALIDAD DE LA EDUCACIÓN EN CHILE}

\section{Introducción y antecedentes}

Existe consenso entre los investigadores en educación acerca de la importancia que tiene un profesor de alto desempeño en los resultados académicos de sus estudiantes. El famoso informe McKinsey enunciaba en el año 2007 que "el techo de un sistema educativo es la calidad de sus profesores". Esta afirmación refleja la importancia que ha tomado el "factor docente" en el debate acerca de las vías para el mejoramiento de la calidad de la educación y señala el desafío de mejorar el desempeño de los docentes al interior de las salas de clases (Barber y Mourshed, 2007).

En el mundo de la investigación educativa no hay consenso acerca de las características que hacen que un profesor sea efectivo. No obstante, se ha demostrado que elevados resultados académicos o alto logro en las pruebas de ingreso a la universidad son características que se asocian de forma decidida con un buen desempeño en los procesos de enseñanza-aprendizaje (Goldhaber, 2008). Siguiendo esta lógica, países como Singapur, Corea del Sur y Finlandia, que permanentemente figuran entre los primeros puestos de pruebas estandarizadas internacionales, están reclutando a sus futuros docentes desde el tercio superior de cada generación, procurando seleccionar a los más aptos (Auguste, Kihn y Miller, 2010).

La evidencia internacional indica que los componentes críticos que atraerían a las personas con mejores resultados académicos a la docencia serían la valoración social de la profesión y la obtención de una buena recompensa económica, al inicio y durante el ejercicio de la carrera (Bellei y Valenzuela, 2010). En la realidad nacional la profesión docente está desvalorizada y las debilitadas condiciones salariales y laborales incidirían en que estudiantes de elevado desempeño académico no quieran desarrollarse profesionalmente en esta área. 


\section{Valoración social de los profesores}

Diversos estudios dan cuenta de una baja valoración social de la profesión docente. Encuestas de percepción realizadas por Adimark GfK y Elige Educar dan como resultado que en diciembre de 2011 solo $35 \%$ de la población consideraba que "el nivel de los docentes en Chile es cada vez mejor", y 24\% consideraba que "los profesores en Chile son respetados"2. Asimismo, un estudio realizado a jóvenes de tercero y cuarto medio, provenientes de los colegios con mejores puntajes promedio en la Prueba de Selección Universitaria, PSU, de la Región Metropolitana, arroja que el 66\% de ellos sitúa a pedagogía como una de las tres carreras con menor prestigio. Junto con ello, solo 22\% considera que "es un orgullo ser profesor en Chile" y solo $15 \%$ cree que "el nivel de los profesores es cada vez mejor" (BID y Elige Educar, 2011).

Sumado a lo anterior, la encuesta "La profesión docente en Chile: políticas, prácticas y proyecciones" entrega como resultado que cerca del $80 \%$ de los profesores declara que el estatus de su profesión es medio o bajo (Ávalos y Sevilla, 2010). Asimismo, la "VIII Encuesta a Actores del Sistema Educativo 2010" da cuenta que el 83,3\% está de acuerdo con que en los últimos 30 años ha existido una desvalorización social progresiva de la profesión docente, y solo $22,3 \%$ de los profesores estima que la sociedad valora sinceramente su profesión (CIDE, 2011).

Existen diversos elementos asociados a la profesión que afectarían la baja valoración social observada: la calidad de formación inicial, las remuneraciones y las condiciones laborales (Cabezas y Claro, 2011).

Respecto de la formación inicial docente se ha observado, entre 1999 y 2009, un aumento del 349\% de la matrícula en carreras de educación (Mineduc, 2011), Según diversos análisis, esto habría

2 Encuesta telefónica realizada por Adimark GFK/Elige Educar en diciembre 2011 a una muestra probable con selección aleatoria de hogares y de entrevistados. El tamaño de la muestra es de 1.114 casos, hombres y mujeres mayores de 18 años de los principales centros urbanos de las 15 regiones del país. El error muestral se estima de 3\% con 95\% de confianza. Para más detalle ver Adimark Gfk/Elige Educar (2011). 
incidido en la calidad de los postulantes que ingresan a dichas carreras, por una creciente flexibilización en los puntajes de corte ${ }^{3}$ (Scheele y Brunner, 2009; Bellei y Valenzuela, 2010). Por otra parte, son las carreras que logran menos años de acreditación (55\% tienen acreditación por tres años o menos) en comparación con otras áreas (Panel de Expertos, 2010).

Los resultados de las pruebas internacionales y nacionales también son indicadores de la calidad de la formación inicial docente. La prueba INICIA 2010 arroja que los egresados de pedagogía responden, en promedio, $46 \%$ de respuestas correctas en la prueba de conocimientos pedagógicos y solo 1\% responde más del 75\% de la prueba correctamente. Por su parte, el estudio TEDS-M de la IEA, que evalúa en el ámbito internacional la formación de los docentes de matemática, arrojó que los futuros profesores del primer ciclo básico en Chile se ubican en el lugar 15 (de 16 países evaluados) y los de segundo ciclo básico en el último lugar (Babcock et al., 2010).

En cuanto a las remuneraciones y estructura de incentivos de los profesores, se observa que sus salarios, a pesar de que se han triplicado desde 1990 a la fecha, se mantienen significativamente inferiores a los de los otros profesionales, comparables con sueldos de carreras técnicas de dos años de duración (Valenzuela et. al 2010). Lo anterior, junto con la existencia de una estructura de incentivos relativamente uniforme y plana, influye de forma directa en las perspectivas salariales y en consecuencia en la desmotivación por elegir educar (Cabezas y Claro, 2011).

Por último, las condiciones laborales de los profesores, asociadas a altos niveles de estrés por situaciones de violencia escolar y por el escaso tiempo para planificar clases, han contribuido también a la baja valoración social de los profesores por parte de la población.

En este contexto desfavorable hacia la profesión surge Elige Educar con el objetivo de contribuir a revertirlo, mejorando la imagen

3 El promedio de puntajes en la Prueba de Selección Universitaria (PSU) de los que ingresaron el año 2009 a alguna carrera en el área de educación fue de 523 puntos, puntaje que corresponde al 34\% de respuestas correctas. Fuente: Índice 2010. 
social del profesor como actor principal en la educación e incentivando el ingreso de buenos candidatos a carreras de pedagogía.

\section{Estrategia de Elige Educar}

El proyecto Elige Educar surge el año 2009 inspirado en la experiencia desarrollada en Inglaterra por el organismo estatal Training and Development Agency for Schools (TDA, Agencia para el Entrenamiento y el Desarrollo de las Escuelas), el cual, mediante una estrategia comunicacional y un programa de incentivos, logró en cinco años que pedagogía se ubicara en el segundo lugar del ranking de valoración social, después de medicina ${ }^{4}$.

En Chile, la idea se anidó en la Facultad de Educación de la Pontificia Universidad Católica de Chile. Conscientes de que este era un desafío del sistema educativo en su conjunto, y que, por lo tanto, requería convocar a otros actores y universidades, se invitó al Centro de Políticas Públicas UC a incubar y transformar esta idea en un emprendimiento. Así se creó Elige Educar, constituyéndose en una alianza público-privada que busca poner fin a la desvalorización social del profesor y convertir la carrera docente en una alternativa profesional atractiva.

Los primeros en adherirse fueron empresas (actuales socios estratégicos), el Ministerio de Educación, la Fundación Chile y el Banco Interamericano de Desarrollo. Así también se sumaron universidades, invitándolas a tener un rol protagónico en la implementación, diseño y desarrollo de estrategias. Esta es una de las principales áreas de trabajo de Elige Educar, pues contribuye a que el mismo sistema se haga cargo de la calidad de los docentes de nuestro país.

Para lograr el impacto esperado trabaja sobre la base de tres ejes estratégicos, los que se detallan en la figura 1.

4 Disponible en www.tda.gov.uk 
Figura 1. Ejes estratégicos de Elige Educar
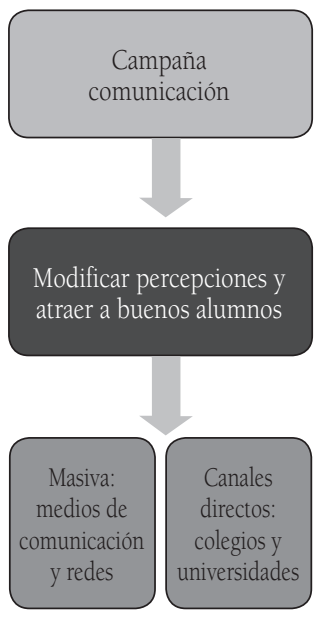

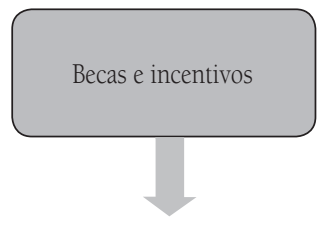

Desarrollar e informar incentivos que aumentan probabilidad Elegir Educar

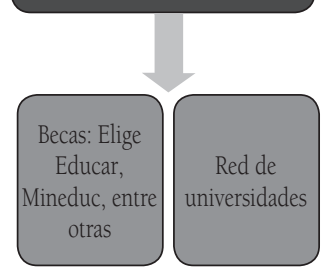

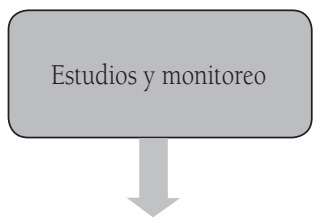

Analizar valoración social de profesores e impactos de Elige Educar

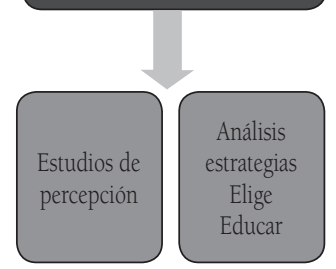

Siguiendo los elementos del Informe McKinsey, Elige Educar organiza su trabajo en tres áreas: atraer, formar y retener buenos profesores al interior del sistema educativo. El área de atracción es la más relevante para la iniciativa, sin embargo se están realizando acciones e impulsando un trabajo interinstitucional cuyo objetivo es afectar de forma indirecta las otras dos áreas.

\section{Atracción de buenos candidatos para ser profesores}

Las estrategias de Elige Educar se han centrado en atraer a jóvenes que presentan condiciones para convertirse en buenos profesores. Siguiendo la experiencia de TDA en Inglaterra y la evidencia disponible, se decidió que las estrategias comunicacionales se destinarían a los jóvenes de mejor rendimiento académico (del tercio superior de sus generaciones, medido por los resultados de la PSU).

Respecto del mensaje para atraer a los jóvenes, el estudio realizado con estudiantes de tercero y cuarto medio, ya mencionado, revela que las principales características que hacen de esta una profesión poco atractiva son: bajos salarios, percepción de que el trabajo es rutinario, estar sometido a situaciones de violencia y/o estrés 
y que es una profesión que brinda pocas oportunidades de desarrollo. Con estos antecedentes, las estrategias se han centrado en modificar estos elementos asociados a la imagen de los profesores, difundiendo testimonios que dan cuenta de lo contrario.

En concreto, las principales actividades que ha desarrollado Elige Educar en esta área, en conjunto con universidades, se detallan a continuación.

\subsection{Intervenciones en colegios}

Elige Educar realiza intervenciones en colegios cuyo objetivo es fidelizar con el proyecto a los distintos actores que conviven en un centro educacional. Los objetivos de las charlas, y a quiénes están dirigidas, se detallan en la figura 2.

Figura 2. Objetivos transversales intervenciones en colegios
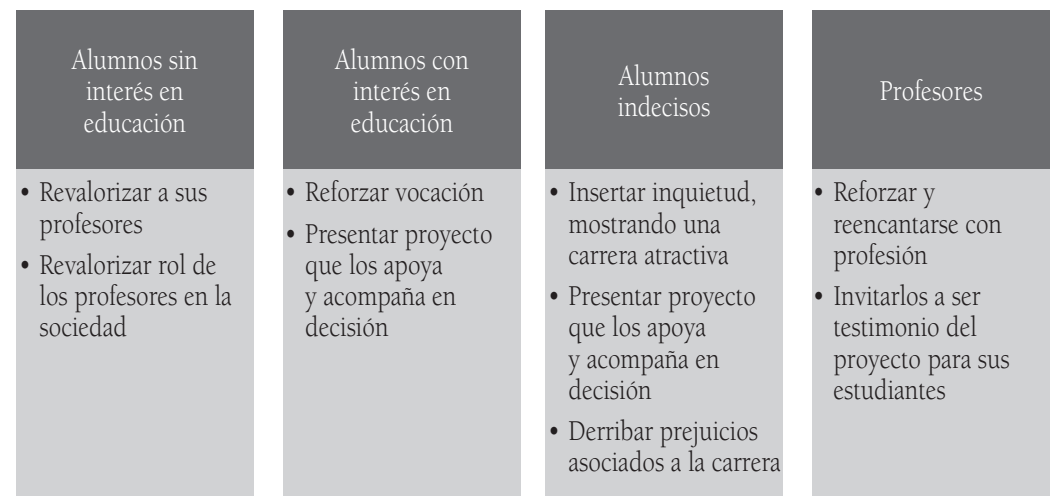

Entre septiembre del 2010 y marzo del 2012 se han realizado intervenciones en 301 colegios y han participado aproximadamente 11.473 estudiantes de tercero y cuarto medio de diversos colegios, de siete regiones del país. Las actividades han sido desarrolladas por estudiantes de educación, seleccionados en conjunto con sus universidades, quienes mediante su testimonio y presentación del proyecto, han incentivado a los jóvenes a conocer la profesión docente, rescatar la importancia del rol y aspectos positivos de los profesores y el valor de la carrera de pedagogía. Esta posibilidad ha permitido 
el encuentro entre estudiantes de carreras de educación de diversas universidades, lo que ha generado un intercambio de experiencias y ha enriquecido el trabajo en los colegios.

\subsection{Ferias vocacionales para apasionados por la educación}

Desde el 2010 a la fecha se han desarrollado siete "ferias para apasionados por la educación" con el objetivo de entregar información de las oportunidades existentes para interesados en estudiar carreras de educación. A ellas han asistido un total aproximado de 1.500 personas (estudiantes escolares, universitarios, profesores y otros profesionales). Cada una de ellas ha contado con la participación y colaboración de universidades, expertos en educación y el Ministerio de Educación. Además se ha invitado a profesores y directores de colegios para que, mediante su testimonio, se genere un espacio de diálogo respecto del rol de los profesores, los desafíos para mejorar su formación y las oportunidades e incentivos que se están generando. Las universidades han sido fundamentales en la difusión y organización de la actividad y han participado dando a conocer sus programas de pregrado y posgrado en educación.

\subsection{Generación de incentivos: Becas Fundación Futuro-Elige Educar}

Para fomentar que los alumnos de buen rendimiento ingresen a carreras de educación Elige Educar, en conjunto con Fundación Futuro, entrega becas a licenciados, egresados o titulados de diversas carreras que se encuentren en el 50\% mejor de su generación, para que estudien programas cortos y diurnos de formación pedagógica en educación media.

Estas becas son una experiencia innovadora dentro del sistema educativo, pues el proceso de diseño e implementación de ellas se realizó conjuntamente con los decanos, coordinadores de programas de media y académicos de las universidades asociadas a ellas ${ }^{5}$.

5 Las universidades que recibieron las becas fueron; P. Católica de Chile, De Chile, Diego Portales, Finis Terrae, De los Andes y Austral de Chile. 
Se han entregado 179 becas distribuidas en diversas especialidades: $40 \%$ en el área de las ciencias (biología, química, matemática y física), 47\% en el área de humanidades (lenguaje, historia y filosofía) y $12 \%$ en inglés. Por otro lado, $85 \%$ de los becarios actualmente egresados se han incorporado al sistema educativo (municipal o particular subvencionado).

\section{Formación de profesores}

A continuación se detallan las estrategias que Elige Educar está desarrollando para generar condiciones que mejoren la calidad de los programas de formación inicial, en conjunto con quienes tienen esta responsabilidad: las universidades.

\subsection{Red de Universidades por la Educación}

Durante el 2011 Elige Educar ha impulsado el programa "Red de Universidades por la Educación”, cuyos objetivos son estimular el ingreso de los alumnos a las instituciones que conforman la red e impulsar y difundir las mejoras en los programas de educación impartidos por ellas.

Ya existen 21 universidades ${ }^{6}$ que se han adherido a esta iniciativa (y 4 se encuentran en proceso de incorporación), comprometidas a impulsar la difusión y desarrollo de políticas de mejora continua en los procesos de reclutamiento, formación e inducción de sus alumnos de educación. Elige Educar, por su parte, se compromete a poner a disposición de las facultades sus medios de comunicación masiva (tradicionales y online), de modo de difundir los avances que implementen las instituciones.

Con este programa, se propicia que se activen procesos sistemáticos de mejora en las universidades. El desafío es constituir una red en que se intercambie información, experiencias e ideas innovadoras entre las instituciones participantes.

6 Universidades De la Frontera, Católica de Temuco, Austral de Chile, San Sebastián, Pontificia Católica de Valparaíso, De Valparaíso, Playa Ancha, De Atacama, Católica del Norte, Católica del Maule, Pontificia Católica de Chile, Alberto Hurtado, Silva Henríquez, De los Andes, Finis Terrae, Diego Portales, Del Desarrollo, De Chile, De Magallanes, De Santiago de Chile y Mayor. 


\subsection{Procesos de selección de estudiantes}

Las becas Fundación Futuro-Elige Educar han sido un mecanismo que permite al proyecto insertarse al interior de los procesos de postulación y selección de las universidades asociadas a la beca. Durante el 2010 se diseñó un proceso de selección de los becarios basado en los modelos usados por Singapur y Finlandia. De este modo se buscó reclutar a los mejores alumnos de cada generación que tuvieran habilidades blandas necesarias para ser profesor. El proceso constó de tres etapas indicadas en la figura 3.

Figura 3. Diagrama de proceso de selección

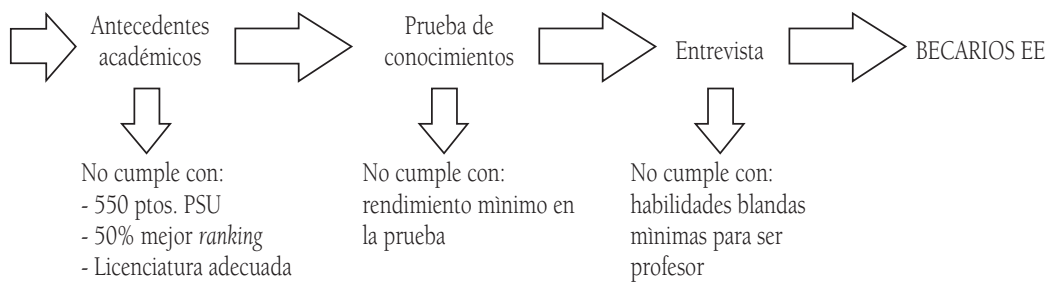

Este ejercicio permitió complejizar y ordenar los procesos de selección al interior de las universidades, especialmente en aquellas que no contaban con un diseño previo basado en criterios objetivos. Elige Educar ha estado incentivando que este proceso se extienda a todos los interesados en postular a las carreras de educación.

\subsection{Inducción de profesores}

Las becas Fundación Futuro-Elige Educar también han sido un instrumento para generar diálogo entre las universidades respecto de los procesos de inducción de los estudiantes que egresen de la carrera. Las becas exigen a los estudiantes trabajar un año en colegios vulnerables con buenas prácticas. Por ello, se está diseñando el plan de inducción y acompañamiento de los becarios y definiendo la red de colegios. Si bien se constituirá un plan específicamente para los estudiantes becarios, Elige Educar está propiciando que el proceso de reflexión sea incorporado y replicado al interior de cada universidad para todos sus estudiantes. 


\section{Primeros avances y desafíos futuros}

En el último año ha estado presente en la agenda pública el tema de la valoración social de los profesores y la carrera docente. Esto ha sido gracias a la convergencia de iniciativas desde distintos sectores (Panel de Expertos, Beca Vocación de Profesor, ley sobre calidad y equidad de la educación, Sistema de promoción y desarrollo profesional docente del sector municipal, entre otras). En este contexto Elige Educar se ha ido constituyendo como una iniciativa que posiciona el tema y que convoca e invita a las universidades a trabajar por mejorar la valoración social de los profesores, atraer a buenos candidatos a la carrera e introducir mejoras en los programas de formación inicial de docentes.

La encuesta Adimark-Elige Educar permite intuir que todos los esfuerzos desplegados, tanto por Elige Educar como por otras iniciativas, estarían generando avances favorables en la percepción pública de los profesores en Chile. Los resultados de diciembre 2011 dan cuenta que, al cabo de dos años, ha aumentado en $8 \%$ la población que apoyaría a su hijo(a) si quisiera estudiar pedagogía (de 68\% a $76 \%$ ) y en $10 \%$ quienes estarían interesados en que ellos o sus hijos estudiaran esta carrera (de 48\% a 58\%). Asimismo, se destaca que 72\% de los encuestados estaría de acuerdo con que "los profesores realizan un aporte a la sociedad mayor de que el de cualquier profesión"7.

En cuanto al eje de incentivos, Elige Educar ha estado difundiendo las oportunidades existentes. Los distintos esfuerzos desplegados también estarían mostrando avances respecto del perfil de jóvenes que está eligiendo educar. Al 2011, las becas "Vocación de Profesor", lanzadas por el Ministerio de Educación, habrían tenido como resultado un aumento del 68\% de matriculados en carreras de educación con más de 600 puntos en la PSU, pasando de 1.824 en el proceso de admisión 2010 a 3.066 en las universidades del Consejo de Rectores (Ministerio Secretaría General de la Presidencia, 2011). Por otra parte, la incorporación de las becas Fundación Futuro-Elige Educar habrían tenido como resultado que profesionales con buenos

7 Ver Adimark Gfk/Elige Educar (2011). 
resultados académicos en su formación universitaria, provenientes de las áreas con mayor déficit de profesores, elijan educar.

Consciente de que aún falta mucho por avanzar, Elige Educar tiene como desafío continuar posicionándose, mediante su campaña comunicacional y acciones concretas, como una iniciativa que busca mejorar la calidad de la educación en Chile relevando a los profesores como los actores clave del proceso. Junto con lo anterior, deberá continuar desarrollando estrategias para que los primeros avances visualizados aumenten y perduren en el tiempo. Asimismo, requerirá seguir entregando aportes concretos al proyecto de Carrera Docente del Ministerio de Educación, como lo hizo con su "Propuestas para una Carrera Docente". Por último, deberá desplegar una mayor capacidad de innovar y adelantarse a nuevas formas de movilizar a las universidades y a la sociedad en su conjunto en la búsqueda de atraer, formar y retener a buenos profesores en la sala de clases.

\section{Referencias bibliográficas}

Adimark Gfk/Elige Educar (2011). Índice Elige Educar: Evaluación de Carrera Docente, diciembre 2011. Santiago.

Auguste, B.; Kihn, P.; Miller, M. (2010). Closing the talent gap: attracting and retaining top-third graduates to careers in teaching: an international and market research-based perspective. McKinsey \& Company.

Ávalos, B.; Sevilla, A. (2010). La construcción de la identidad profesional en los primeros años de docencia: evidencia de la investigación. Centro de Investigación Avanzada en Educación (CIAE). Universidad de Chile.

Babcock, J.; Babcock, P.; Buhler, J.; Cady, J.; Cogan, L.; Houang, R.; et al. (2010). Breaking the cycle: an international comparison of U.S. mathematics teacher preparation. Initial findings from the teacher education and development study in mathematics (Teds-M). Center for Research in Math and Science Education, Michigan State University.

Barber, M.; Mourshed, M. (2007). How the world's best-performing school systems come out on top. McKinsey \& Company.

Banco Interamericano de Desarrollo, Elige Educar (2011). Estudio sobre la percepción que tienen los jóvenes de $3^{\circ}$ y $4^{\circ}$ medio sobre las carreras 
de educación, la experiencia laboral docente y la disposición a estudiar pedagogía. Área de Estudios Elige Educar, Santiago.

Bellei, C.; Valenzuela, J.P. (2010) ¿Están las condiciones para que la docencia sea una profesión de alto estatus en Chile? Núcleo Milenio sobre la Profesión Docente de la Universidad de Chile. Centro de Investigación Avanzada en Educación (CIAE). Universidad de Chile.

Beyer, H.; Alvarado, J.; Arellano, JP.; Aylwin, M.; Brunner, J.J.; Krebs, A.; et al. (2010). Propuestas para fortalecer la profesión en el sistema escolar chileno. Panel de expertos para una educación de calidad. MINEDUC.

Cabezas, V.; Claro, F. (2011). Valoración social del profesor en Chile: ¿cómo atraer a alumnos talentosos a estudiar pedagogía? Centro de Políticas Públicas UC. Temas de la Agenda Pública. Año 6, n. ${ }^{\circ}$ 42, P. Universidad Católica de Chile.

Centro de Investigación y Desarrollo de la Educación, CIDE (2011). VIII Encuesta a Actores del Sistema Educativo 2010. Universidad Alberto Hurtado de Chile.

Goldhaber, D. (2008). "Teachers Matter, but Effective Teacher Quality is Elusive". En Hellen F. Ladd y Edward B. Fiske (eds.), Handbook of Research in Education Finance and Policy (pp. 146-165). New York: Routledge.

Ministerio de Educación (2011). Presentación Resultados prueba Inicia Egresados Pedagogía en Educación Básica 2010. Disponible en www. mineduc.cl

Ministerio de Educación (2010). Resultados Evaluación Docente 2009. Disponible en www.acreditaciondocente.cl

Ministerio Secretaría General de la Presidencia (2011). Reporte de Cumplimiento de los Siete Ejes Prioritarios del Gobierno del Presidente Sebastián Piñera. Unidad Presidencial de Gestión del Cumplimiento, División de Coordinación Interministerial. Santiago.

Scheele, J.; Brunner, J. J. (2009). Educación terciaria y mercado laboral. Formación profesional, empleo y empleabilidad. Una revisión de la literatura internacional. Futuro Laboral, División de Educación Superior del Ministerio de Educación, Santiago, Chile.

Valenzuela, J.P.; Sevilla, A.; Bellei, C.; De los Ríos, D. (2010). Remuneraciones de los docentes en Chile: resolviendo una aparente paradoja. Serie Documentos de Orientación para Políticas Públicas, 2. Núcleo Milenio sobre la Profesión Docente en Chile. Centro de Investigación Avanzada en Educación (CIAE). Universidad de Chile. 
248 ELIGE EDUCAR, UN PUNTO DE ENCUENTRO ENTRE ACTORES PARA MEJORAR LA CALIDAD DE LA EDUCACIÓN EN CHILE - M. P. Medeiros, G. Gutiérrez, H. Hochschild, R. Lira

\section{Bibliografía recomendada}

Sanders, W.L.; Rivers, J.C. (1996). Cumulative and residual effects of teachers on future student academic achievement. Knoxville: University of Tennessee Value-Added Research and Assessment Center. Tennessee.

Recibido: 01/04/2011

Aceptado: 17/05/2012 